\title{
Surface Modification of Asymmetric Polysulfone/Polyethylene Glycol Membranes by DC Ar-Glow Discharge Plasma
}

\author{
Chalad Yuenyao,, ${ }^{1,2}$ Thawat Chittrakarn, 3,4 \\ Yutthana Tirawanichakul, ${ }^{1,3,4}$ and Suksawat Sirijarukul ${ }^{3,4}$ \\ ${ }^{1}$ National Research University Project 2014, Prince of Songkla University, Hat Yai, Songkhla 90112, Thailand \\ ${ }^{2}$ Material Chemistry Research Center, Department of Chemistry, Faculty of Science, Khon Kaen University, Muang, \\ Khon Kaen 40002, Thailand \\ ${ }^{3}$ Department of Physics, Faculty of Science, Prince of Songkla University, Hat Yai, Songkhla 90112, Thailand \\ ${ }^{4}$ Membrane Science and Technology Research Center, Faculty of Science, Prince of Songkla University, Hat Yai, \\ Songkhla 90112, Thailand \\ Correspondence should be addressed to Chalad Yuenyao; pannatorn.y@hotmail.com
}

Received 1 September 2016; Revised 9 November 2016; Accepted 29 November 2016

Academic Editor: Mohd H. D. Othman

Copyright (c) 2016 Chalad Yuenyao et al. This is an open access article distributed under the Creative Commons Attribution License, which permits unrestricted use, distribution, and reproduction in any medium, provided the original work is properly cited.

Polysulfone/polyethylene glycol (PSF/PEG) membranes were prepared by dry/wet phase inversion method. Effects of direct current glow discharge plasma using argon as working gas on morphological structures and gas separation properties of membranes were studied. Alteration of membrane characteristics were analyzed by various techniques like contact angle, scanning electron microscope, Fourier transform infrared spectroscopy, and dynamic mechanical thermal analysis. Gas separation properties were measured in terms of permeation and ideal $\mathrm{O}_{2} / \mathrm{N}_{2}$ selectivity. Results showed that hydrophilic and gas separation properties of PSF/PEG membranes increased by plasma surface modification. It was also shown that the dosage of PEG and plasma treatment affected the morphological structures and mechanical and gas separation properties. The macro voids and transmembrane structure disappeared with a little amount of PEG dosage. Pore size and mechanical strength tend to decrease with increasing PEG dosage up to $10 \mathrm{wt} \%$. Glass transition temperature $\left(T_{g}\right)$ receded from 201.8 to $143.7^{\circ} \mathrm{C}$ for pure PSF and PSF/PEG with PEG dosage of $10 \mathrm{wt} \%$. $\mathrm{O}_{2}$ and $\mathrm{N}_{2}$ gases permeation through the 10-minute plasma treated membranes tend to increase. However, the permeation strongly dispersed when treatment time was more extended.

\section{Introduction}

Plasma surface modification is one of the processes with high potential that can be used to raise the utility of membrane materials in diverse technical applications. Glow discharge plasma at low pressures is one of the several physical techniques utilized to modify the morphology and properties of polymeric membrane surfaces [1]. Most glow discharge plasmas used for these aims are generated from radio frequency $(13.56 \mathrm{MHz})$ and microwave $(2.45 \mathrm{GHz})$ power sources. However, DC glow discharge is the easiest among these techniques as there is no need for complex electronic circuits. As already presented by various references, polymeric membrane modification is the integration of chemical and physical techniques [2-4]. Addition of organic or inorganic particle as a third component of the casting solution is one of the most important chemical techniques, while the plasma surface modification is a physical (plasma treatment) and physicochemical (plasma polymerization and grafting) technique. Membrane performance obtained by the incorporation of these two techniques is quite high [5]. Typically, the permeability of asymmetric polymeric gas separation membranes prepared by phase inversion techniques is quite low and unsuitable for practical use. In order to increase the permeability of gas, hydrophilic properties, selective layer thickness, and porosity of the membranes have to be improved. In general, to increase the permeation rate, the selective layer should be thin. For the $\mathrm{CO}_{2} / \mathrm{CH}_{4}$ separation, the hydrophilic property of the membrane surface has an influence on the $\mathrm{CO}_{2}$ permeation rate. Therefore, 
the permeation rate of $\mathrm{CO}_{2}$ was enhanced by increasing the hydrophilic property of the membrane surface $[3,4]$. However, the increase of gas permeation rate depends on many parameters like kinetic diameter, quadrupole moment, and polarizability of gas, as well as the polarity of polymeric membrane surfaces. Generally, etching and deposition processes occur at the same time when the polymeric membranes are treated with the discharge plasma from non-polymer forming gases [1]. Nevertheless, these processes are depended on the initial plasma conditions.

For the addition of the third component in the polymer solution, polyethylene glycol (PEG) is one of the most important additives used. Several research groups have investigated the effect of PEG and both molecular weight and dosage, on the morphology, structure, mechanical properties, and performance as well as the formation of membranes. In 1998, Kim and Lee [5] studied the effect of PEG on membrane formation by phase inversion. They found that the PEG behaves as a pore-forming agent. The water flux increases and solute rejection and coagulation value decrease with increasing PEG to NMP ratio. Also, porosity of the bottom sublayer increased with the use of the PEG additive. Besides, the free fractional volume is uplifted as the glass transition temperature $\left(T_{g}\right)$, the modulus and tensile strength declined after PEG was incorporated in the casting solution $[6,7]$. Shieh et al. [7] found that PEG can be used to improve the membrane selectivity due to its hydrophilic nature. Chakrabarty et al. [8] reported that the membranes which incorporated PEG of higher molecular weights show higher pure water flux and porosity than the membranes with low molecular weight PEG. Zuo et al. [9] investigated the influence of PEG molecular weight on morphologies and properties of PVDF membranes. They found that PEG with a relatively low molecular weight acts as a pore-forming agent while PEG with high molecular weight behaves as a pore-suppressing agent. They also found that the viscosity of casting solutions increased with increasing PEG molecular weight. Furthermore, Yunos et al. [10] investigated the effect of PEG molecular weight and dosage on the properties and performance of PSF membranes. They found that pure water flux and mean pore radius of membranes increased with an increase of PEG content as well as the hydrophilic component appearance in PSF/PEG blend membranes.

This work studied the effects of PEG as a polymeric additive and of low temperature and pressure DC glow discharge plasma on the morphological structures and surface properties of polysulfone/polyethylene glycol (PSF/PEG) membranes. Additionally, the permeation of $\mathrm{N}_{2}$ and $\mathrm{O}_{2}$ gases through PSF/PEG membranes before and after plasma treatment was measured and appraised.

\section{Experimental Method}

2.1. Materials. Polysulfone (UDEL 3500 LCD MB) in pellet form was supplied from Solvay, China. Solvents, including DMAC $\left(99 \%, M_{W}=87.12 \mathrm{~g} / \mathrm{mol}\right), 1$-methyl-2-pyrrolidone (NMP), tetrahydrofuran (THF), and acetone (99.5\%), were purchased from Fluka Riedel-deHaën, Sigma-Aldrich (Thailand) Co., Ltd., and Ajax Finechem Pty Ltd., respectively. PSF pellets were weighed by $0.01 \mathrm{~g}$ weighing scale (AND and GF3000) from A\&D (Thailand) Co., Ltd. A hot plate magnetic stirrer of VS-130SH from Vision Scientific (Thailand) Co., Ltd., was used for dissolving PSF. Hot plate temperature was set at $55-60^{\circ} \mathrm{C}$. PEG $\left(M_{W}=1,000 \mathrm{~g} / \mathrm{mol}\right)$ purchased from Fluka Riedel-deHaën was used as additive in this study.

2.2. Preparation of Flat Sheet Membranes. Polysulfone/polyethylene glycol (PSF/PEG) asymmetric membrane was prepared by dry/wet phase inversion method. Pellets of PSF were dried by an electrical oven at $85^{\circ} \mathrm{C}$ for $24 \mathrm{hr}$ before usage. All ingredients were weighed corresponding to the given formula. In the preparation of the casting solution, PEG was first dissolved in the solvents. Afterwards, PSF materials are carefully poured into the solution of PEG and solvents. To remove air bubbles, the dissolved casting solutions were subjected to the ultrasonic bath for $15 \mathrm{~min}$. Then, the obtained casting solution was casted on a clear and smooth glass plate. The wet thickness of casted membranes was controlled at about $150 \mu \mathrm{m}$. To obtain the asymmetric membrane, the nascent membranes were placed in the normal air for one minute before they were immersed in the coagulation bath of ionized water for $24 \mathrm{hr}$. After that, the membrane was dried in normal air for $24 \mathrm{hr}$.

2.3. Plasma Treatment of PSF/PEG Membranes. A lab-scale low pressure DC glow discharge plasma system was used for the treatment of PSF/PEG membranes. Details of this plasma system are explained in previous work [11]. In brief, the plasma system as shown in Figure 1 consisted of vacuum chamber, power supply, and electrode and rotary pump. Before generating plasma discharges, the pressure inside the plasma reactor was pumped down to about $5.5 \times 10^{-2}$ mbar. In the present work, the initial plasma gas pressure and discharge power were controlled at about $2.5 \times 10^{-1}$ mbar and 15.0 W, respectively. Samples of PSF/PEG membranes were fixed on an acrylic plate of $1.5 \mathrm{~mm}$ thickness and then placed on the anode electrode. The interelectrode gap was controlled at about $3.0 \pm 0.1 \mathrm{~cm}$. To study the effect of glow discharge plasma on the surface and gas permeation properties of PSF/PEG membranes, plasma treatment time was varied.

2.4. Characterization of PSF/PEG Membranes. First, PSF membranes with different PEG concentrations were tested for their hydrophilic properties by water contact angle measurements in sessile drop mode using a video based optical contact angle measuring instrument (Model OCA 15EC, DataPhysics Intruments $\mathrm{GmbH}$, Germany). Samples of plasma treated PSF/PEG membranes were subjected to water contact angle measurements as well. Physical and mechanical properties of polymeric membrane were investigated by dynamic mechanical thermal analysis (DMTA) technique in tensile mode. During the DMTA test, the frequency and temperature were controlled at $1 \mathrm{~Hz}$ and $20-300^{\circ} \mathrm{C}$, respectively. For the observation of morphological patterns and structures by SEM, samples of PSF/PEG membranes were immersed in liquid nitrogen for 5-10 minutes and then coated by gold at low pressure. Furthermore, the creation of functional groups 


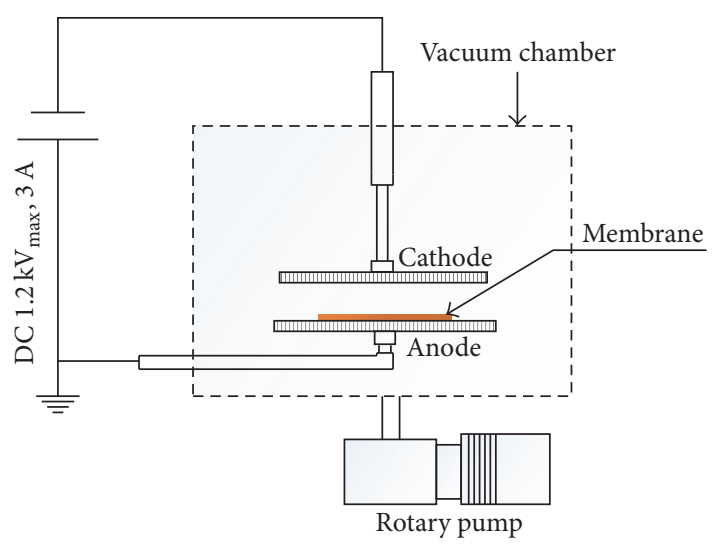

FIGURE 1: DC-plasma system and components.

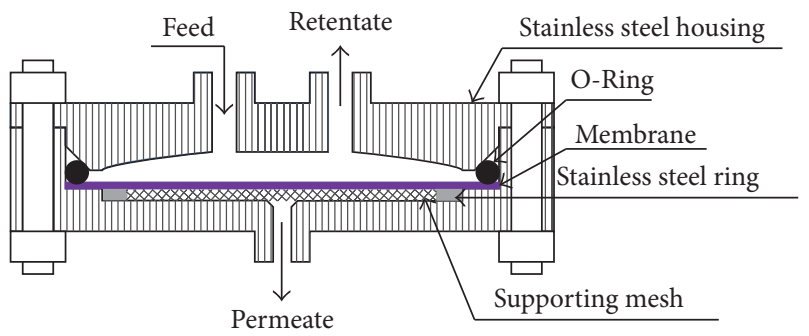

FIGURE 2: Components of the permeation module.

was verified by attenuated reflectance Fourier transform infrared (ATR-FTIR) spectroscopy in the range of 400$4,000 \mathrm{~cm}^{-1}$ for the wave number with a resolution of $4 \mathrm{~cm}^{-1}$.

2.5. Gas Permeation Measurement. The permeation rate of pure $\mathrm{O}_{2}$ and $\mathrm{N}_{2}$ gases through PSF/PEG membrane samples before and after plasma treatment was measured at different pressure levels, at 2, 4, 6, and 8 bar. The permeation module as a critical component of the testing setup is presented in Figure 2. Samples of membrane were cut into circular form with the diameter of $5.7 \mathrm{~cm}$ and effective area of about $17.36 \mathrm{~cm}^{2}$. To prevent any leakage of gas, rubber O-rings with a thickness of $0.5 \mathrm{~cm}$ were utilized. Permeation rates were read from the soap bubble flow rate meter, five times for each sample, with the average value calculated. The pressurenormalized flux or permeability value in the unit of GPU $\left(1 \mathrm{GPU}=10^{-6} \mathrm{~cm}^{3}\right.$ (STP) $/ \mathrm{cm}^{2} \mathrm{~s} \mathrm{~cm} \mathrm{Hg}$ ) was determined by using (1) [4, 12-14]. In addition, the ideal gas separation factor or the $\mathrm{O}_{2} / \mathrm{N}_{2}$ selectivity was estimated by $(2)[3,10,14]$.

$$
\begin{aligned}
\left(\frac{P}{l}\right) & =\frac{Q}{A \Delta p} \\
\propto_{i j} & =\frac{(P / l)_{i}}{(P / l)_{j}}=\frac{P_{i}}{P_{j}},
\end{aligned}
$$

where $Q$ is the volumetric flow rate, $P$ is the permeability, $l$ is the skin layer thickness, $A$ is the membrane effective area, $(P / l)$ is the permeance in the unit of GPU, and $\Delta p$ is the transmembrane pressure. $P_{i}$ and $P_{j}$ are the permeability of $i$ and $j$ gases, respectively.

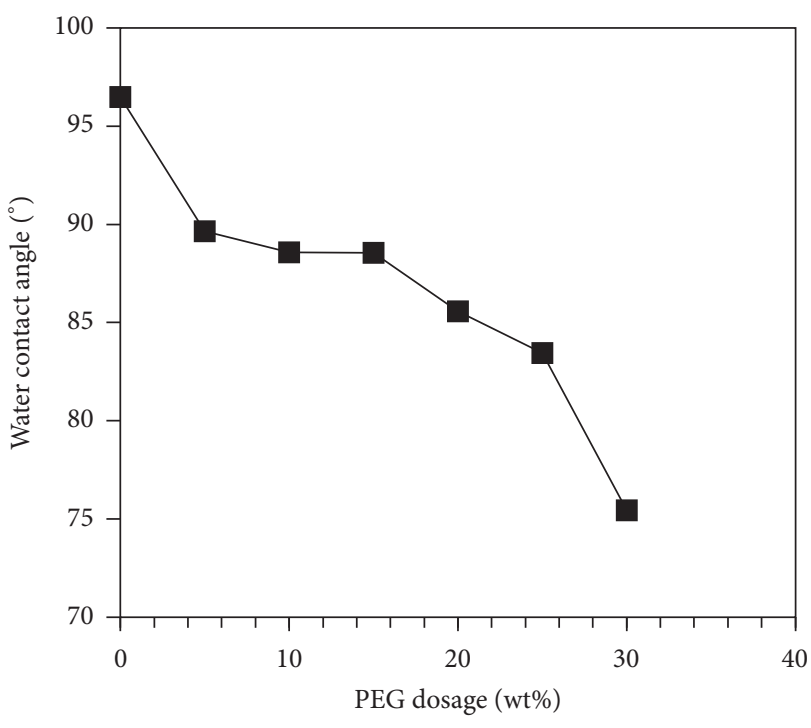

FIGURE 3: Water contact angle versus an increase in the PEG concentration (wt\%).

\section{Results and Discussion}

3.1. Effect of PEG Concentration. Results of the water contact angle measurement of PSF membranes at different PEG dosages show a decreasing trend, especially when the PEG concentration increased to $30 \mathrm{wt} \%$ as shown in Figure 3. The water contact angle decreased from 90.5 to 75.4 or about $17.0 \%$ while the PEG dosage increased to about $30 \mathrm{wt} \%$. This means that the PEG concentration has an effect on the hydrophilic properties of PSF membranes.

Figures 4(a)-4(c) show morphological patterns and structures of pure PSF, PSF/PEG $5 \mathrm{wt} \%$, and PSF/PEG $10 \mathrm{wt} \%$, respectively. It can be seen that the macro voids disappeared when PEG was incorporated in the PSF membrane. In this study, the pore size was estimated through the SEM micrograph. It was found that the average pore size of PSF membranes approximated by Carnoy Program 2.0 ${ }^{\circledR}$ version incorporated with SEM micrographs tends to decrease with increasing PEG dosage. The average pore size of pure PSF (included macro voids), PSF/PEG $5 \mathrm{wt} \%$, and PSF/PEG $10 \mathrm{wt} \%$ was about $3.37,3.11$, and $3.09 \mu \mathrm{m}$, respectively. Further, it can be seen that the small transition layer which appeared in the pure PSF membrane was completely suppressed when PEG was incorporated. Figure 5 shows the pore diameter distribution of pure PSF membranes and PSF/PEG membranes with a loading of 5 and $10 \mathrm{wt} \%$. Distribution of the pore size is narrower when PEG $\left(M_{W}=1,000\right.$ dalton) was incorporated in the casting solution. This means that the pore size of PSF/PEG membranes is more uniform than that of pure PSF ones.

In addition to the hydrophilic properties and morphological structures, mechanical strengths and glass transition temperatures $\left(T_{g}\right)$ of PSF membranes were studied using DMTA technique. Results show that mechanical strengths in terms of storage modulus and loss modulus of the membrane samples tend to decrease while the PEG dosage increased up to $10 \mathrm{wt} \%$ 


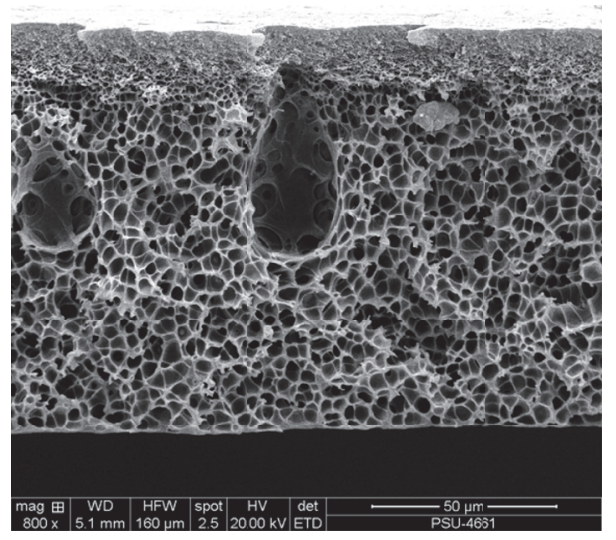

(a)

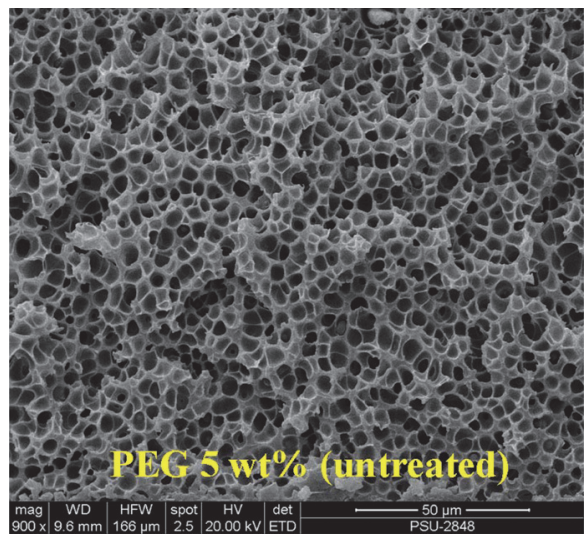

(b)

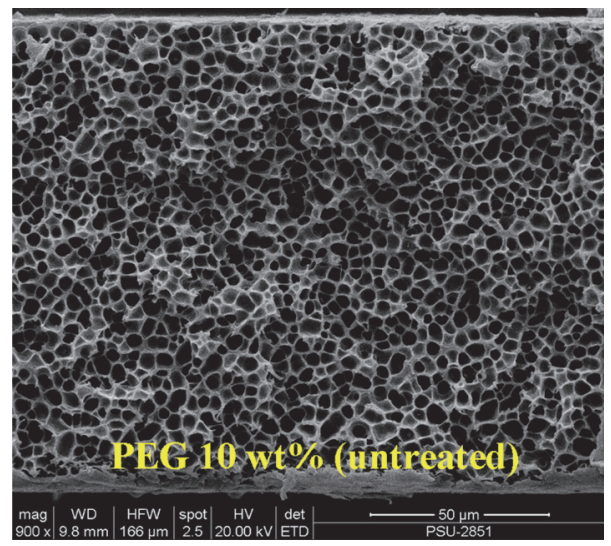

(c)

FIGURE 4: SEM micrographs: cross-sectional structure of pure PSF (a), PSF/PEG 5 wt\% (b), and PSF/PEG 10 wt\% (c) membranes.

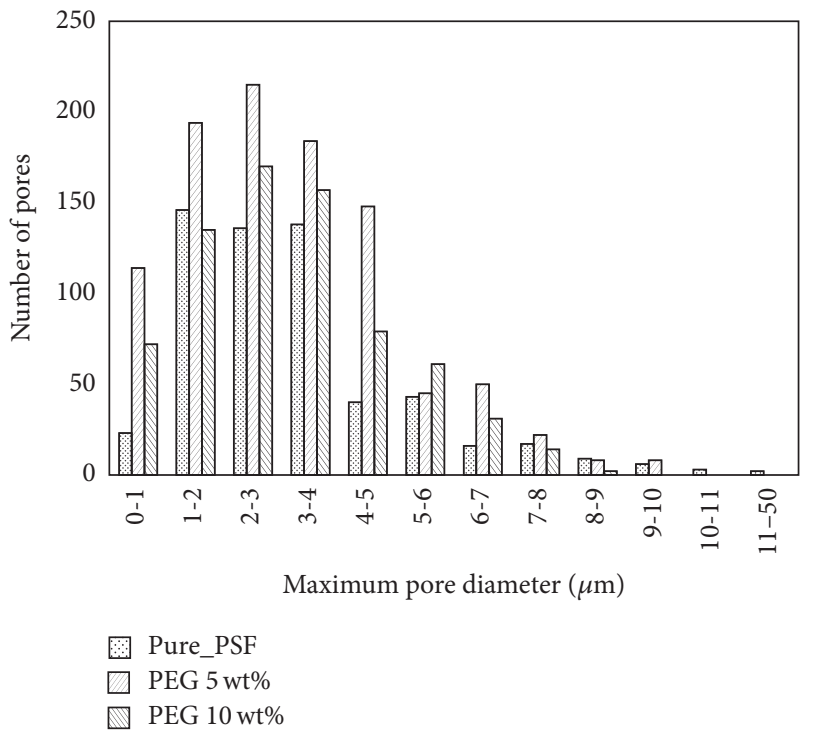

FIGURE 5: Comparison of the maximum pore diameter distributions between pure PSF membranes and PSF/PEG membranes with a loading of 5 and $10 \mathrm{wt} \%$, respectively. as illustrated in Figures 6(a) and 6(b), respectively. Figure 6(c) shows that $T_{g}$ decreased from $201.8^{\circ} \mathrm{C}$ for a membrane without PEG to $143.7^{\circ} \mathrm{C}$ for a PSF/PEG membrane with $10 \mathrm{wt} \%$ PEG. Decreasing of mechanical strengths and $T_{g}$ may reflect the degradation of membrane structure caused from plasma treatment and the negative alteration influenced from aggregation of PEG dosage. These results are in accordance with Kim and Lee [5], Ma et al. [15], and Chakrabarty et al. [16].

3.2. Effect of DC-Plasma Treatment. In addition to the effect of PEG dosage, Ar-plasma also has an effect on the physical properties of PSF/PEG. The storage and loss modulus of plasma treated PSF/PEG membranes decreased as shown in Figures 6(a) and 6(b). This indicates that Ar-plasma at about $15 \mathrm{~W}$ can degrade PSF/PEG membranes. Figure 7 shows the modification of water contact angle of PSF/PEG membranes (PEG concentration of 5 and $10 \mathrm{wt} \%$ ) both before and after plasma treatment at about $15 \mathrm{~W}$ and at different treatment times. It can be seen that the water contact angle of PSF/PEG membrane surfaces decreased sharply at the beginning of the treatment and dispersed for a prolong treatment time. These changes indicated a competition between etching and 


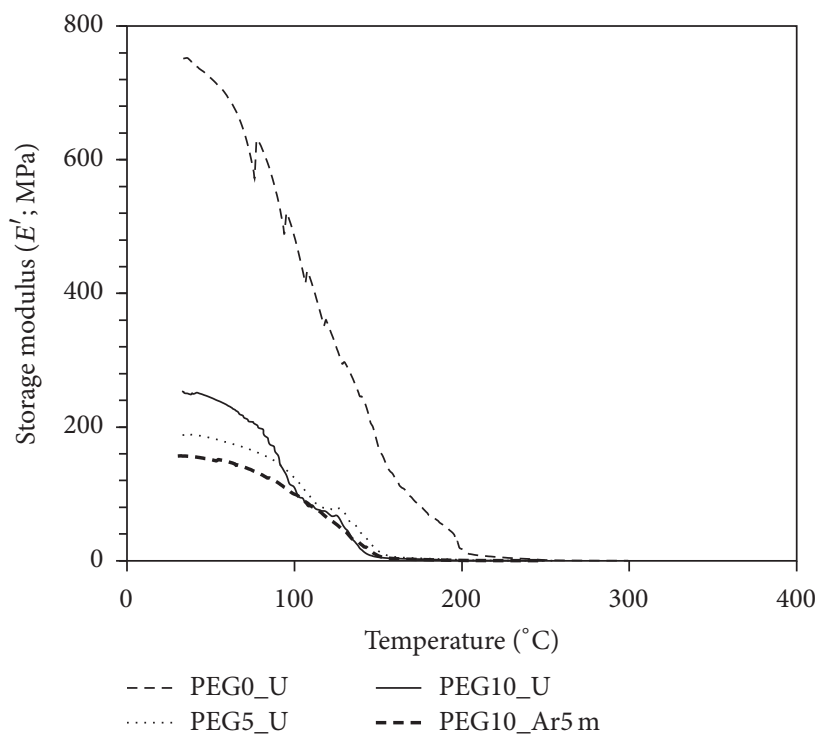

(a)

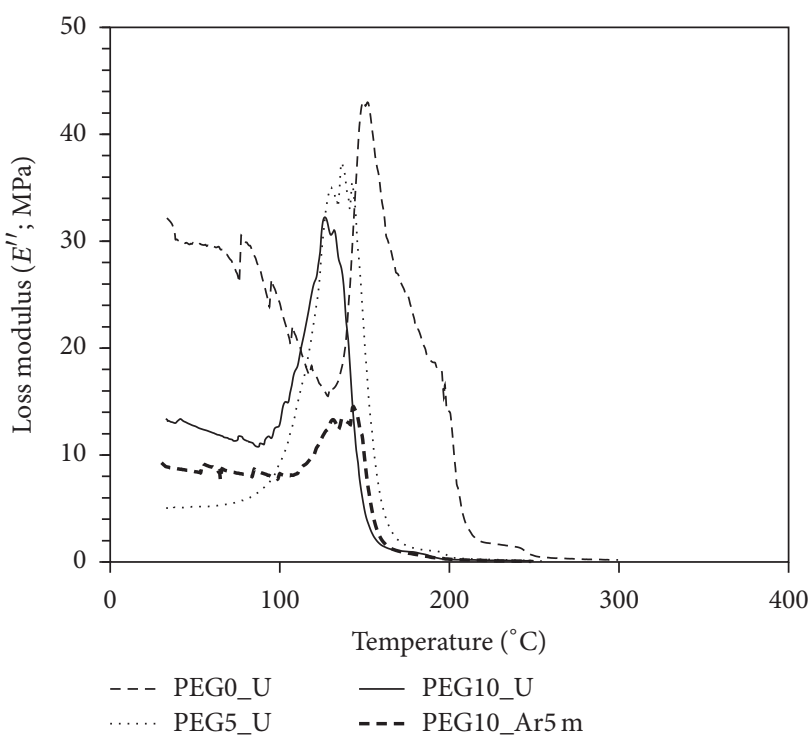

(b)

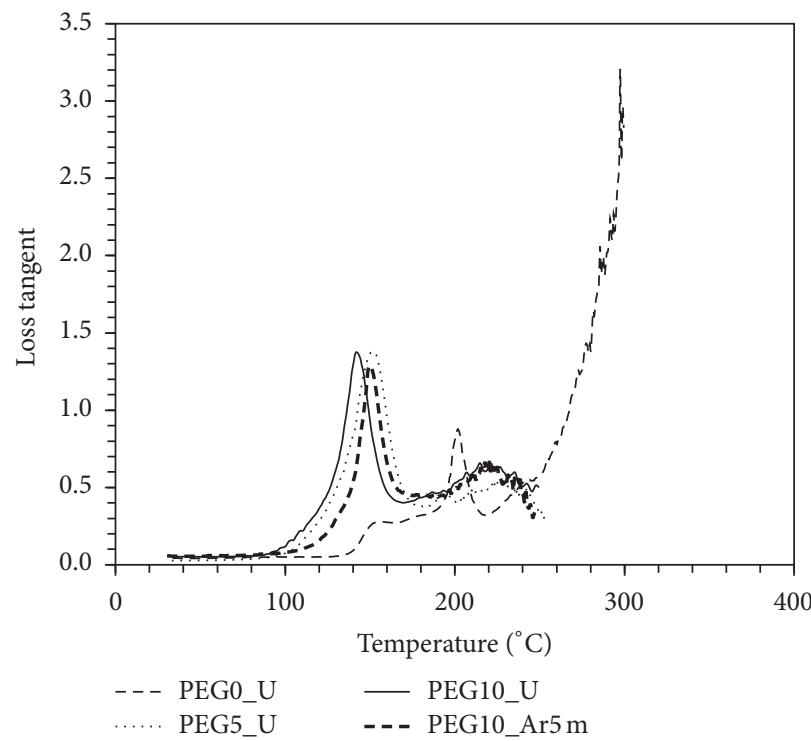

(c)

FIGURE 6: Variation of storage and loss modulus, loss tangent, and $T_{g}$ of membrane samples with PEG concentration (wt\%).

deposition processes in the plasma treatment. For a prolong treatment time, the etching effect and deposition process are comparable. For this treatment, the optimized plasma treatment time is in the range of 1-3 min. It was shown that low pressure DC Ar-plasma can improve the hydrophilic properties of PSF/PEG membrane surfaces. From literature, the improvement of the hydrophilic property of polymeric membrane surfaces can increase both permeation rate and ideal selectivity of $\mathrm{CO}_{2} / \mathrm{CH}_{4}$ [3].

Figure 8 shows the FTIR spectra of PSF/PEG membranes before and after Ar-plasma treatment. For the PSF/PEG untreated membranes, it can be seen that the characteristic absorption peaks of PEG at around $2,885 \mathrm{~cm}^{-1}(\mathrm{O}-\mathrm{H})$ and $1,110 \mathrm{~cm}^{-1}$ (C-O) do not appear. This suggested that most of the PEG is not apparent in the membrane top layer. However, a low absorption peak appeared at around 2,872, 2,920, and $2,965 \mathrm{~cm}^{-1}$. After Ar-plasma treatment, the new low absorption peak appeared at around 2,357 and $2,368 \mathrm{~cm}^{-1}$. Also, very low absorption peaks at around $3,140-4,000 \mathrm{~cm}^{-1}$ are in the opposite direction when compared with the PSF/PEG untreated membranes. The intensity of absorption peaks at around 2,872 and $2,965 \mathrm{~cm}^{-1}$ decreased after plasma treatment whereas the peak at around $2,920 \mathrm{~cm}^{-1}$ increased clearly. Decreasing of absorption peaks may reflect the decomposition or degradation of functional groups whereas the increase of absorption bands was attributed to the deposition or creation of functional groups.

New absorption peaks correspond to the creation of $\mathrm{C}-\mathrm{H}$ bonds of aldehyde groups. After plasma treatment, membrane samples were exposed to the air and the oxidation 


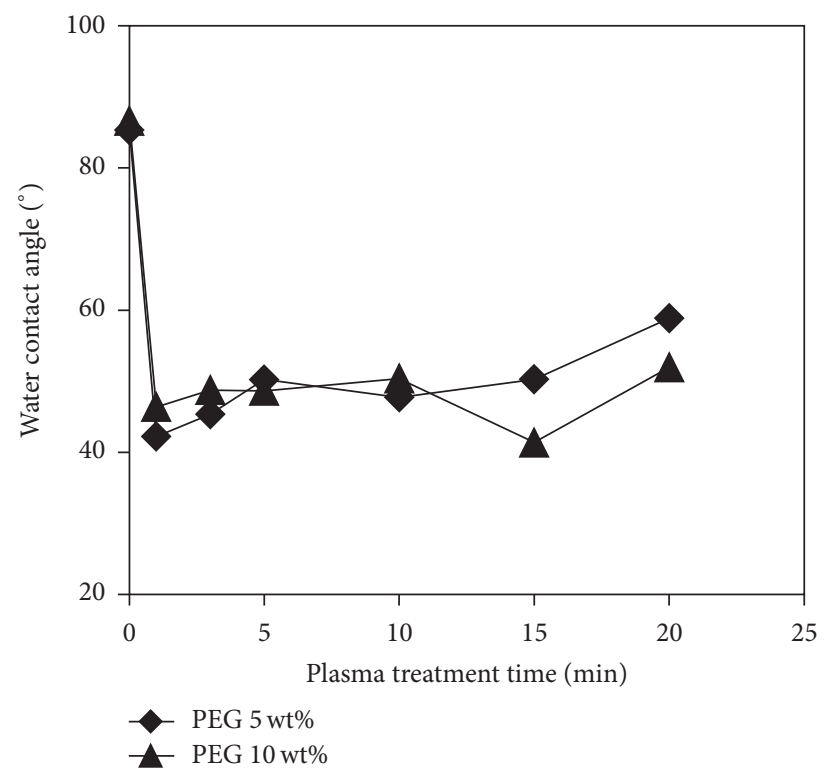

FIGURE 7: Water contact angle of PSF/PEG membranes after being treated with low temperature DC Ar-plasma at treatment time of $0,1,3,5$, 10,15 , and $20 \mathrm{~min}$.

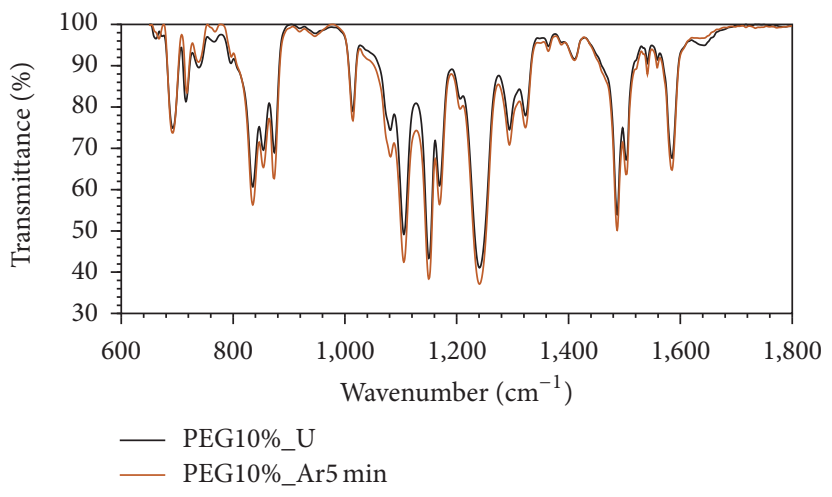

(a)

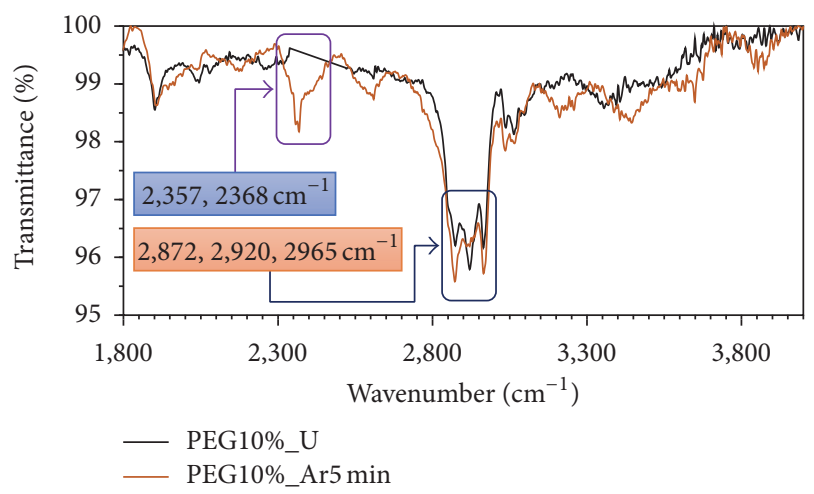

(b)

FIGURE 8: FTIR spectra of PSF/PEG membranes before (black) and after (orange) plasma treatment; (a) wavenumber 600 to 1,800 and (b) 1,800 to 4,000 .

of methyl group in the structure of PSF was then initiated. The oxidation at the position of methyl group yields an aldehyde which can further be oxidized to yield a carboxylic polar group. The increase of hydrophilic properties may be the result from these polar groups. Furthermore, the O$\mathrm{H}$ and $\mathrm{C}-\mathrm{H}$ bonds at around 3,000 to $3,900 \mathrm{~cm}^{-1}$ band of plasma treated membranes are more active than the untreated ones. For this reason, the hydrophilic property was improved according to the results of the water contact angle measurements mentioned above.

Figure 9 shows the top skin surface of PSF/PEG $10 \mathrm{wt} \%$ membranes. It can be seen that the number of pores decreased after plasma treatment. This may due to the effect that the deposition processes are more dominant than the plasma etching processes. This result is according to the gas permeation testing results, as the permeation of $\mathrm{O}_{2}$ and $\mathrm{N}_{2}$ gases through PSF/PEG $10 \mathrm{wt} \%$ decreased after being treated by plasma. This leads to an increase of the ideal $\mathrm{O}_{2} / \mathrm{N}_{2}$ selectivity.
3.3. Gas Permeation Property. The permeation of $\mathrm{O}_{2}$ and $\mathrm{N}_{2}$ gases through PSF and PSF/PEG membranes was measured using different transmembrane gas pressures. The ideal $\mathrm{O}_{2} / \mathrm{N}_{2}$ separation factor was evaluated. The experimental results of these measurements are shown in Figure 10. It can be seen that the permeance in the unit of GPU of $\mathrm{O}_{2}$ and $\mathrm{N}_{2}$ is strongly dispersed. However, the permeance tends to decrease with increasing plasma treatment time. The gas permeation through ten-minute plasma treated membranes increased clearly. This can be explained that the plasma etching process is more dominant than the deposition process. The variation of $\mathrm{O}_{2}$ and $\mathrm{N}_{2}$ permeability affected the ideal separation factor or selectivity of these two gases. The ideal $\mathrm{O}_{2} / \mathrm{N}_{2}$ selectivity increased to about 3 as shown in Figure 10 when PSF/PEG membranes were treated with glow discharge plasma of Ar gas for 20 minutes. Results from this study also showed that the gas permeation of PSF/PEG membranes is significantly higher than that of PSF pure 


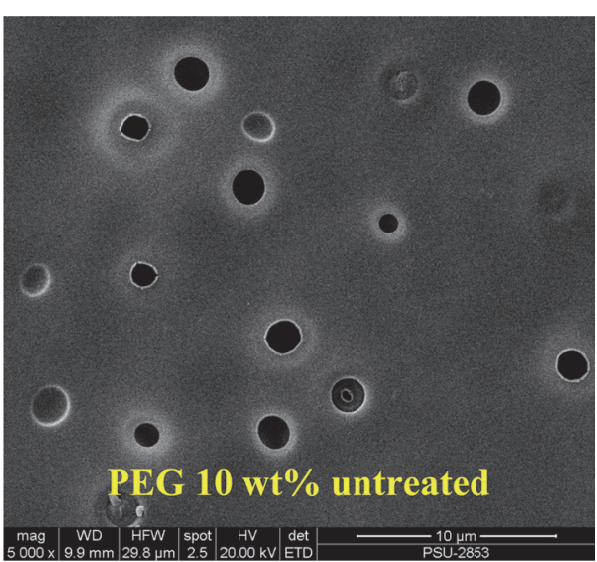

(a)

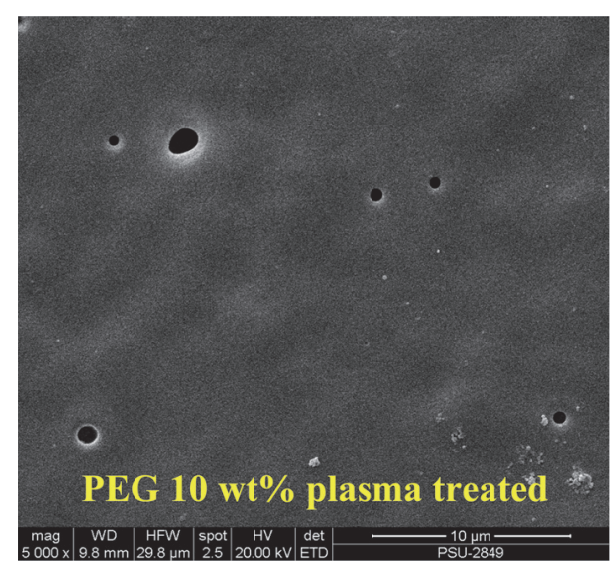

(b)

FIGURE 9: SEM micrographs of top skin layers of PSF/PEG $10 \mathrm{wt} \%$ membranes, untreated (a) and Ar-plasma treated at a discharge power and treatment time of $15 \mathrm{~W}$ and 5 min (b), respectively.

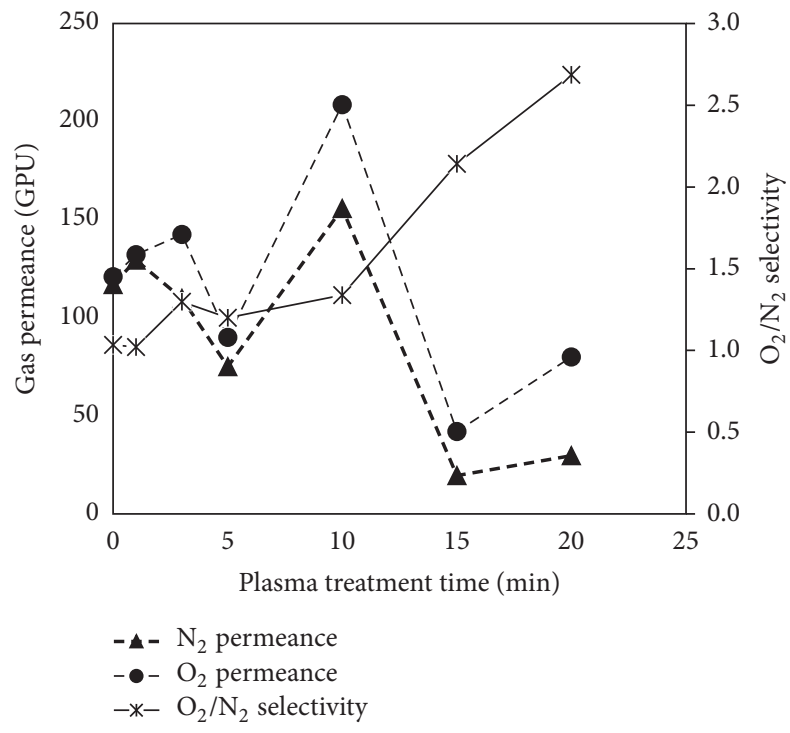

FIGURE 10: $\mathrm{O}_{2}$ and $\mathrm{N}_{2}$ gases permeance and ideal $\mathrm{O}_{2} / \mathrm{N}_{2}$ selectivity at a pressure of 3 bar for plasma treated PSF membrane with different treatment times.

membranes. For the asymmetric polymeric membrane with dense skin layer, the permeation of gas depended on the skin layer thickness. Referring to the SEM micrographs as shown in Figures 4(a)-4(c), the skin layer thickness decreased when PEG was incorporated in PSF membranes. Typically, skin layer thickness affected to the permeation rate of gases [4]. Furthermore, incorporation of PEG into the PSF casting solution affected the formation of porous top layer as shown in Figure 9. Formation of pore on top skin layer leads to increase of permeation rate of gases and decrease of selectivity of $\mathrm{O}_{2} / \mathrm{N}_{2}$ gases.

\section{Conclusions}

In summary, the morphological structure and physical property of PSF changed when PEG was incorporated in the casting solution. The pore size distribution was optimized clearly. Macro voids were suppressed and instead sponge like structures appeared. The hydrophilic properties were improved with incorporation of PEG. The level of hydrophilicity was enhanced with increasing PEG dosage. In addition to the change of morphological structures, the physical properties in terms of glass transition temperature and viscoelastic modulus were modified. The hydrophilic property was further increased by glow discharge plasma at low pressures, because the polar group like $\mathrm{O}-\mathrm{H}$ and $\mathrm{C}-\mathrm{H}$ bonds increased after plasma treatment. For the gas separation property, it was shown that the incorporation of PEG can increase the permeation rate of $\mathrm{O}_{2}$ and $\mathrm{N}_{2}$ gases. However, the $\mathrm{O}_{2} / \mathrm{N}_{2}$ separation factor is quite low. This might be because the coupling agent is not incorporated in the casting solution. The separation factor was enhanced after PSF/PEG membranes were treated with DC glow discharge plasma.

\section{Competing Interests}

The authors declare that they have no competing interests.

\section{Acknowledgments}

Financial support from National Research University, Prince of Songkla University (NRU'58, PSU), and ThEP Center, CHE, Thailand, is acknowledged. The authors wish to thank the Membrane Science and Technology Research Center (MSTRC), Department of Physics, and Prince of Songkla University for providing infrastructure and facilities required.

\section{References}

[1] E. F. Castro Vidaurre, C. A. Achete, F. Gallo, D. Garcia, R. Simão, and A. C. Habert, "Surface modification of polymeric materials by plasma treatment," Materials Research, vol. 5, no. 1, pp. 37-41, 2002. 
[2] D. Hegemann, H. Brunner, and C. Oehr, "Plasma treatment of polymers for surface and adhesion improvement," Nuclear Instruments and Methods in Physics Research, Section B: Beam Interactions with Materials and Atoms, vol. 208, pp. 281-286, 2003.

[3] S. Modarresi, M. Soltanieh, S. A. Mousavi, and I. Shabani, "Effect of low-frequency oxygen plasma on polysulfone membranes for $\mathrm{CO}_{2} / \mathrm{CH}_{4}$ Separation," Journal of Applied Polymer Science, vol. 124, no. 1, pp. E199-E204, 2012.

[4] C. Yuenyao, Y. Tirawanichakul, and T. Chittrakarn, "Asymmetric polysulfone gas separation membranes treated by low pressure DC glow discharge plasmas," Journal of Applied Polymer Science, vol. 132, no. 24, article no. 42116, 2015.

[5] J.-H. Kim and K.-H. Lee, "Effect of PEG additive on membrane formation by phase inversion," Journal of Membrane Science, vol. 138, no. 2, pp. 153-163, 1998.

[6] G. D. Vilakati, E. M. V. Hoek, and B. B. Mamba, "Probing the mechanical and thermal properties of polysulfone membranes modified with synthetic and natural polymer additives," Polymer Testing, vol. 34, pp. 202-210, 2014.

[7] J.-J. Shieh, T.-S. Chung, R. Wang, M. P. Srinivasan, and D. R. Paul, "Gas separation performance of poly(4vinylpyridine)/polyetherimide composite hollow fibers," Journal of Membrane Science, vol. 182, no. 1-2, pp. 111-123, 2001.

[8] B. Chakrabarty, A. K. Ghoshal, and M. K. Purkait, "Effect of molecular weight of PEG on membrane morphology and transport properties," Journal of Membrane Science, vol. 309, no. 1-2, pp. 209-221, 2008.

[9] D.-Y. Zuo, Y.-Y. Xu, W.-L. Xu, and H.-T. Zou, “The influence of PEG molecular weight on morphologies and properties of PVDf asymmetric membranes," Chinese Journal of Polymer Science, vol. 26, no. 4, pp. 405-414, 2008.

[10] M. Z. Yunos, Z. Harun, H. Basri, and A. F. Ismail, "Studies on fouling by natural organic matter (NOM) on polysulfone membranes: effect of polyethylene glycol (PEG)," Desalination, vol. 333, pp. 36-44, 2014.

[11] C. Yuenyao, T. Chittrakarn, Y. Tirawanichakul, P. Saeung, and W. Taweepreda, "The effects of oxygen and argon plasmas on the surface morphology of polysulfone membrane," Thai Journal of Physics, vol. 8, pp. 41-44, 2012.

[12] A. F. Ismail and P. Y. Lai, "Development of defect-free asymmetric polysulfone membranes for gas separation using response surface methodology," Separation and Purification Technology, vol. 40, no. 2, pp. 191-207, 2004.

[13] M. A. Aroon, A. F. Ismail, M. M. Montazer-Rahmati, and T. Matsuura, "Morphology and permeation properties of polysulfone membranes for gas separation: effects of non-solvent additives and co-solvent," Separation and Purification Technology, vol. 72, no. 2, pp. 194-202, 2010.

[14] R. W. Baker, Membrane Technology and Applications, John Wiley \& Sons, Chichester, UK, 2nd edition, 2004.

[15] Y. Ma, F. Shi, J. Ma, M. Wu, J. Zhang, and C. Gao, "Effect of PEG additive on the morphology and performance of polysulfone ultrafiltration membranes," Desalination, vol. 272, no. 1-3, pp. 51-58, 2011.

[16] B. Chakrabarty, A. K. Ghoshal, and M. K. Purkait, "SEM analysis and gas permeability test to characterize polysulfone membrane prepared with polyethylene glycol as additive," Journal of Colloid and Interface Science, vol. 320, no. 1, pp. 245253, 2008. 

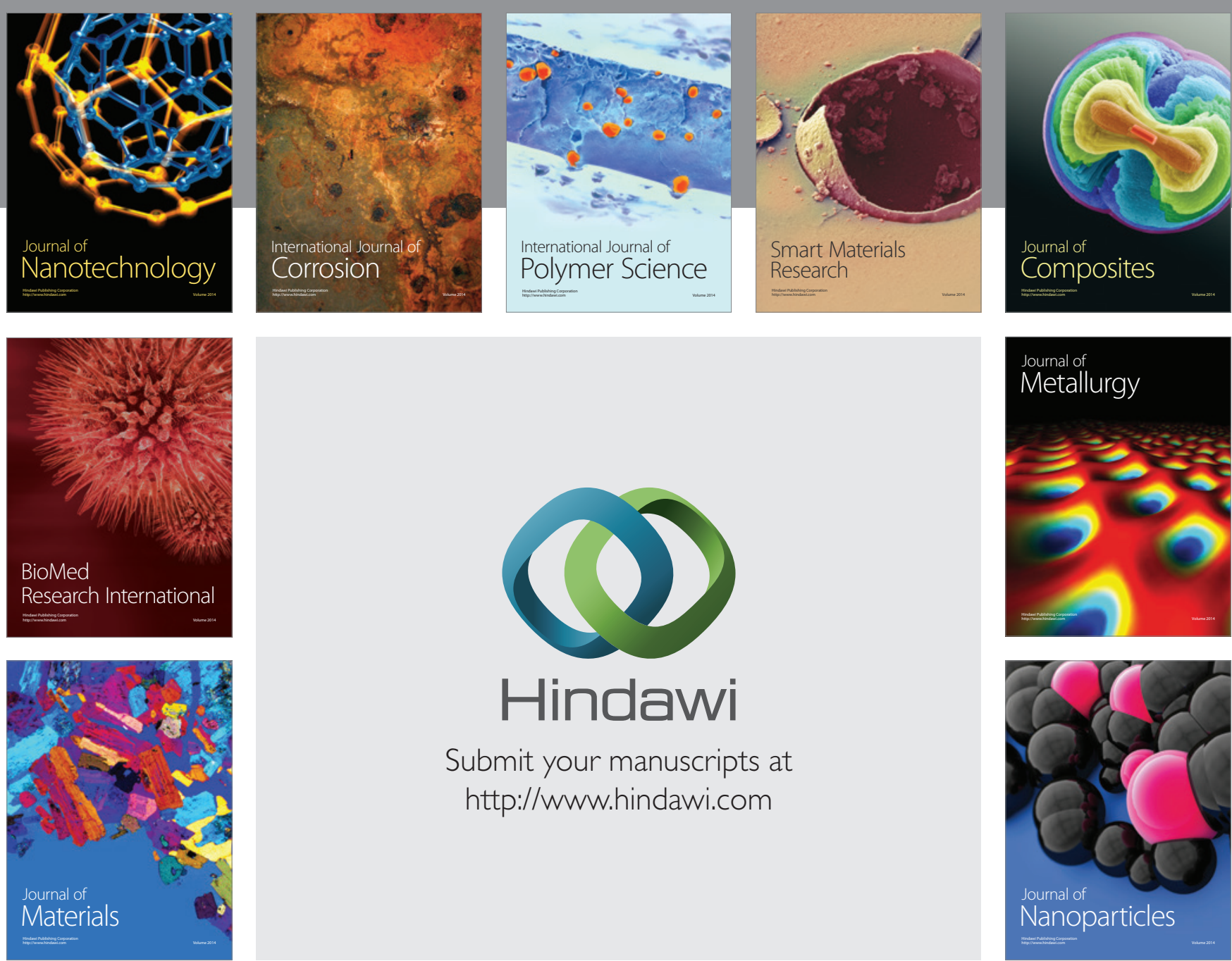

\section{Hindawi}

Submit your manuscripts at

http://www.hindawi.com

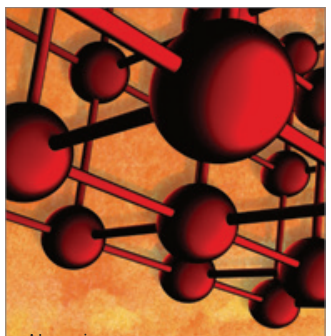

Materials Science and Engineering
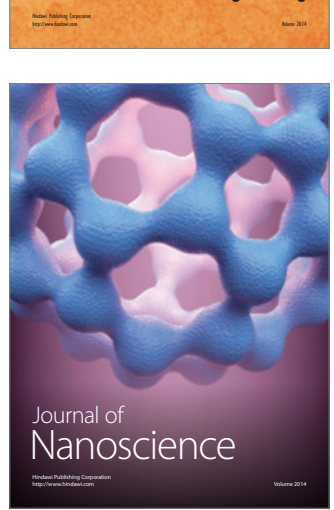
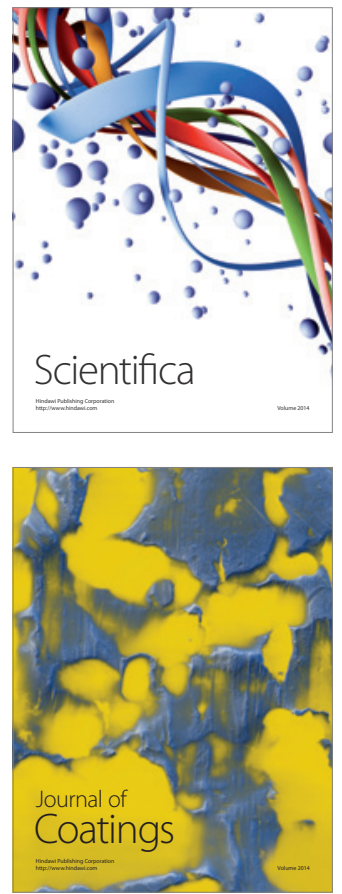
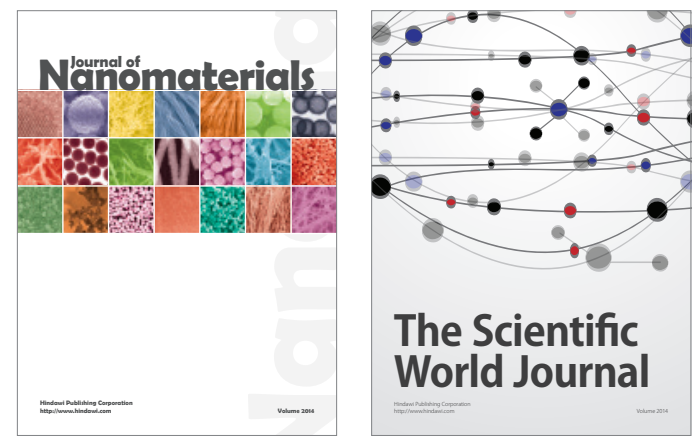

The Scientific World Journal
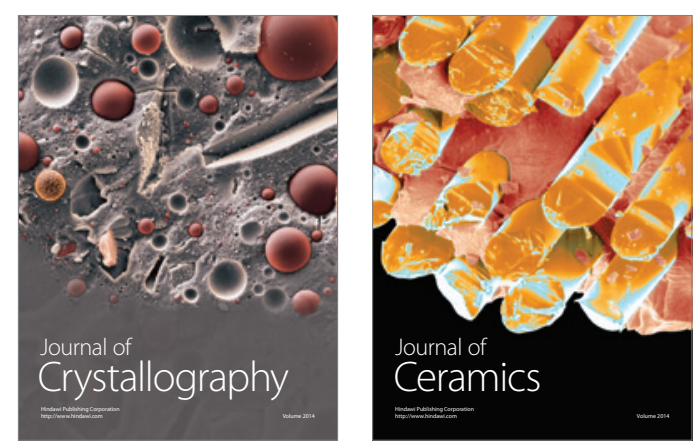
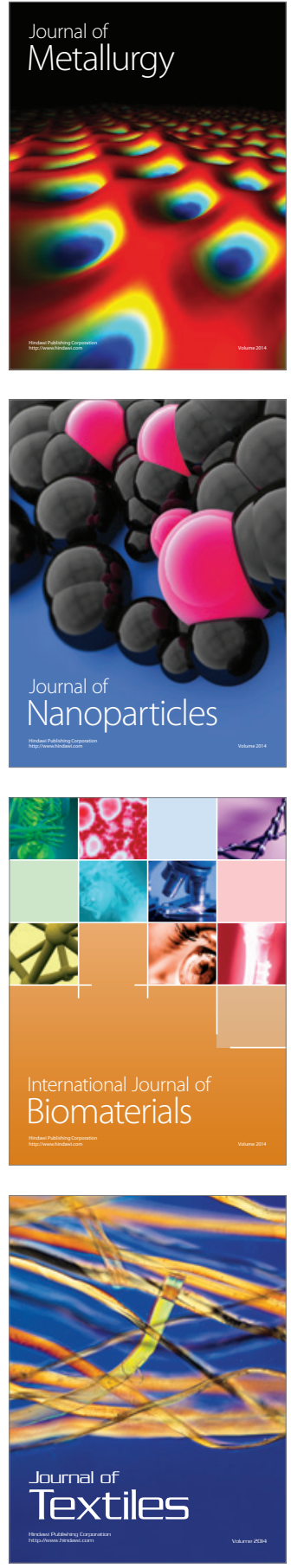